\title{
Escolha de parceiros sexuais e investimento parental: uma perspectiva desenvolvimental
}

\author{
Roberta Tavares de Melo Borrione \\ Eulina da Rocha Lordelo \\ Universidade Federal da Bahia
}

\begin{abstract}
RESUMO
Este artigo apresenta duas teorias acerca da escolha de parceiro(a)s sexuais, assim como suas implicações para a Psicologia do Desenvolvimento. O campo de investigação objetiva explicar os mecanismos envolvidos na escolha de parceiro(a)s sexuais - atração sexual, desenvolvimento de vínculos e estabelecimento de relações duradouras. A Teoria da Estratégia Sexual possui como principal premissa o conceito de estratégia, que considera o comportamento humano como direcionado para objetivos específicos e para solucionar problemas de acasalamento, oriundos do ambiente evolucionário de adaptação da história humana. A adaptação decorre do contexto, especialmente da natureza das estratégias de acasalamento - curto ou longo prazo. Essas estratégias utilizariam o investimento parental como mecanismo psicológico de escolha de parceiro(a)s. Uma abordagem desenvolvimental, sobreposta à primeira, prioriza formação e manutenção de vínculos entre parceiro(a)s sexuais, proveniente de dois fatores: o primeiro constitui-se num mecanismo de escolha exaptado do mecanismo de apego e o segundo baseia-se nas características propensão, familiaridade e enamoramento romântico. Atualmente, não se podem refutar quaisquer dessas visões. Deve-se aguardar respostas conclusivas pelo teste de hipóteses rivais. Em qualquer caso, a teoria deve explicar, simultaneamente, universalidade, diversidade cultural e trajetórias desenvolvimentais particulares sob a perspectiva evolucionária. Nesse empreendimento, devem ser elucidadas dimensões causal, funcional, ontogenética e filogenética.
\end{abstract}

Palavras-chave: escolha de parceiro(a)s sexuais; investimento parental; Psicologia Evolucionista.

\begin{abstract}
Mate Selection and Parental Investment: a Developmental Approach

The purpose of this article is to present two theories about human mate selection, as well as their implications to Developmental Psychology. The field of investigation aims to explain the mechanisms involved in mate selection - sexual attraction, bond development, and the establishment of long-term relationships. The Sexual strategies theory has as its primary scientific assumption the concept of strategy. This concept assumes that human behavior is goal-specific and goal-directed to solve mate problems derived from the evolutionary environment in which human history of adaptation has evolved. Adaptation takes place based upon context, especially upon the nature of mating association strategies - short or long-term relationships. These strategies would have evolved using parental investment as a psychological mechanism of mate selection. Another approach based on a developmental point of view - which overlaps the Sexual strategies theory - emphasizes bond development among sexual partners, derived from the sum of two factors: the first one is a mechanism of selection exaptated from the attachment mechanism, and the second one is based on characteristics of propensity, propinquity, and romantic infatuation. Currently, it is impossible to refute any of these approaches. It is utmost necessary to await conclusive answers, possible only testing rival hypotheses. A conclusive theory should be able to simultaneously explain universality, cultural diversity and specific developmental pathways taking into account an evolutionary approach. In this endeavor, causal, functional, ontogenetic, and phylogenetic dimensions should be enlightened.
\end{abstract}

Keywords: mate selection; parental investment; Evolutionary Psychology.

\section{INTRODUÇÃO}

Este artigo tem o propósito de apresentar a teoria da estratégia sexual sobre a escolha de parceiros sexuais, no seu estado corrente, e uma perspectiva desenvolvimental que se lhe contrapõe, buscando esclarecer as consequiências dessas abordagens para a pesquisa e suas implicações para a Psicologia do Desenvolvi- mento. O campo de investigação busca explicar os mecanismos envolvidos na escolha de um parceiro sexual - atração sexual, desenvolvimento de vínculos e estabelecimento de relações duradouras.

A TEORIA DE ESTRATÉGIA SEXUAL 
A Teoria de Estratégia Sexual possui como principal premissa a concepção de estratégia como inerente aos acasalamentos humanos. O termo estratégia é utilizado para indicar a natureza do comportamento humano de acasalamento em ser direcionado para objetivos específicos e em ser voltado para solucionar problemas, não envolvendo, contudo, ação conscientemente planejada ou articulada (Bjorklund \& Kipp, 1996; Buss \& Schmitt, 1993).

As estratégias dependem do contexto, especialmente do contexto temporal das associações de acasalamento - de curto ou longo prazo - e partem do pressuposto de que homens e mulheres vivenciaram diferentes problemas de acasalamento no ambiente evolucionário de adaptação da história humana (Buss \& Schmitt, 1993). Devem existir, portanto, pelo menos em domínios específicos, diferentes princípios reguladores desse comportamento feminino e masculino, relacionados às estratégias de curto ou longo prazo.

Princípios reguladores do comportamento de acasalamento de homens e mulheres traduzem-se em mecanismos de preferência psicológicos que solucionaram problemas de sobrevivência e reprodução, através de adaptações especializadas, no curso da história evolucionária humana. Em termos gerais, estes podem ser definidos como "(...) processos psicológicos [regras ou algoritmos de processamento de informação (Ellis, 1995)] que inclinam ou predispõem organismos a seletivamente escolher ou rejeitar estímulos em seu ambiente" (Buss, 1995, p. 253). Essas preferências operam como desejos, atrações e emoções, geradoras de sentimentos de proximidade ou repulsa, não sendo, conseqüentemente, conscientes e intencionais (Buss \& Schmitt, 1993), e podem ser tanto passivos - ao rejeitar ou aceitar objetos que são proporcionados - ou ativos - ao mobilizar comportamento em prol ou contra determinados objetos.

Buss e Schmitt (1993), ao analisarem The descendent of man and selection in relation to sex, retomam as considerações de Darwin acerca da evolução: apenas as características promotoras de vantagens reprodutivas persistem, sendo estas avaliadas segundo o sucesso no nível de competição intra-sexual e no nível de atração inter-sexual.

Buss (1995) considera existir influência dos padrões de escolha seletiva sobre as táticas de competição intra-sexual, as quais funcionam como reguladoras internas dos comportamentos.

As pressões de seleção durante o período evolucionário originaram mecanismos, nesse caso, mecanismos de preferência; quando mecanismos de preferência evoluem, eles começam a exercer pressão seletiva sobre outros componentes do acasalamento humano. Mecanismos de preferência expressos por um sexo devem afetar os padrões de competição intra- sexual demonstrados por membros do sexo oposto (Buss, 1995, p. 253).

Como se daria o desenvolvimento desses mecanismos psicológicos de preferência por parceiros?

Kriegman (1999) sugere que tendências a comportamentos específicos ou a capacidade de experienciar afetos sob determinadas situações devem ter sido resultado de vantagens adaptativas moldadas pela seleção natural. Esta seleção geraria funcionamentos psicológicos capazes de promover determinados comportamentos, ainda que eles não fossem passíveis de especificação direta. Os comportamentos originar-seiam de causas distais, isto é, de forças ou pressões seletivas que moldaram um padrão de comportamento, uma motivação ou um processo mental (consciente ou inconsciente) durante o período evolucionário (ou história filogenética). Essas causas distais modelaram, assim, os afetos relacionados a esses comportamentos, submetidos ao reforço seletivo pelos mecanismos do ambiente proximal.

Os mecanismos proximais constituir-se-iam na expressão desses afetos interligados à exibição de comportamentos funcionais ou adaptativos, ou seja, os mecanismos proximais representariam propensões afetivas, que se perpetuaram, por assegurarem um resultado diferencial de maior sucesso reprodutivo (em freqüência) às gerações subseqüentes (Kriegman, 1999).

Pode-se compreender, neste sentido, que não há predições de mecanismos proximais específicos ou de expressões diretas de uma função adaptativa.

Como se compreender, então, o mecanismo de preferência sexual de parceiro(a)s?

Trivers (1972, citado por Buss \& Kenrick, 1998; Buss \& Schmitt, 1993;) considerou como a principal força propulsora da seleção sexual o investimento parental que cada sexo despende na sua prole. Por investimento parental, compreende-se qualquer forma de investimento que os pais (nesse caso, mãe e/ou pai) despendem em favor de uma prole específica, de forma a aumentar a sua chance de sobrevivência e, conseqüentemente, de reprodução, em detrimento de investimento em uma outra prole.

Trivers propõe ainda haver uma forte ligação entre investimento parental e seleção sexual (atração intersexual versus competição intra-sexual). Em outras palavras, o sexo que investe mais na prole seria mais exigente quanto à escolha do(a) parceiro(a) (atração inter-sexual) e o sexo que investe menos competiria mais avidamente em prol da obtenção de membros do sexo oposto "mais investidores", com maior potencial e valor dentro de sua comunidade (competição intrasexual) (Buss \& Kenrick, 1998; Buss \& Schmitt, 1993; Kriegman, 1999). 
Apoiando-se na descoberta do mesmo padrão de comportamento de investimento parental em várias espécies nas quais os papéis sociais são reversos (o macho tipicamente investindo mais na prole do que a fêmea), essa teoria considera que o investimento parental dirige e orienta o processo de seleção sexual, ou seja, a escolha de parceiros ocorre não pelo que este pode representar no momento do ato sexual, mas pelo papel que este poderá desempenhar como pai/mãe de uma futura prole (Trivers, 1972, conforme citado por Buss \& Schmitt, 1993).

Segundo a teoria de Trivers (1972, conforme citado por Buss \& Schmitt, 1993), como a mulher necessariamente deve investir mais tempo e recursos na prole do que o homem (relação de anos - no processo de fertilização, nidação, gestação, lactação e cuidado com a criança por parte da mulher - e mínimo de minutos - simples ato sexual no caso dos homens), o sexo feminino seria o sexo de maior seletividade e de maior discriminação frente a possíveis parceiros de acasalamento (Bjorklund \& Kipp, 1996; Buss, 1995; Buss \& Kenrick, 1998; Buss \& Schmitt, 1993; Kriegman, 1999), constituindo-se, assim, no sexo de maior atração inter-sexual. Já o sexo masculino seria menos discriminativo e exerceria maior competitividade intra-sexual. Mesmo assim, os homens têm grande oportunidade de investir na sua prole desde o nascimento e, quando o fazem, seguindo o princípio do investimento parental em relação à seleção sexual, devem selecionar mais as suas parceiras.

Geary e Flinn (2002) sustentam a existência de mecanismos sexuais diferenciados (inclusive apoiados em manifestações hormonais) no desenvolvimento e na manifestação do investimento parental. Estes se constituiriam em estratégias reprodutivas originadas diversamente entre homens e mulheres, segundo respostas a problemas adaptativos, oriundos de relacionamentos e alianças temporais intra e inter-sexuais e de situações promotoras de estresse. A proteção e o cuidado da prole - tidos como formas de investimento parental - e a formação e o estabelecimento de redes de relações de favorecimento (befriending) ocorreriam de forma diferenciada a depender do objetivo e da função do sexo do indivíduo e estariam reciprocamente interligados. Assim, a forma diferenciada de investimento parental na sua prole deve ter gerado, em milhares de gerações, mecanismos diferenciados de seleção de parceiros para acasalamento.

Postula-se ainda a existência de diferenças sexuais no padrão de inibição de comportamentos em tarefas sociais, comportamentais e cognitivas, no contexto evolucionário, nos quais as mulheres sofreram maiores pressões em prol da inibição de determinados comportamentos, devido ao desenvolvimento e à manifestação de atividades relacionadas à reprodução e à educação/criação de crianças, os quais, em conse- qüência, expressam a existência de diferenças sexuais no investimento parental (Bjorklund \& Kipp, 1996).

Como se pode compreender, então, a relação entre investimento parental e mecanismos de preferência sexual de parceiro(a)s? Pode-se vislumbrar a existência de uma forte ligação entre investimento parental e seleção sexual como estratégias sexuais diferenciadas, sejam estas de curto ou longo prazo. Na verdade, a durabilidade e o objetivo dos encontros entre parceiros serão marcados por estratégias de seleção diferenciadas. Nesse sentido, para se compreender a relação entre investimento parental e estratégias sexuais de seleção diferenciada entre sexos, é necessário abordar as diversas formas de associações entre parceiros, segundo critérios de curto ou longo prazo (Buss \& Kenrick, 1998; Buss \& Schmitt, 1993).

\section{Estratégias sexuais de curto prazo}

Historicamente, os benefícios reprodutivos para homens que utilizam uma estratégia sexual de curto prazo podem ser facilmente encontrados: há maior probabilidade de gerar maior prole através do aumento de parceiras sexuais do que pelo aumento do número de filhos por parceira (Buss \& Schmitt, 1993). O sucesso reprodutivo do homem reside na sua capacidade de inseminar um maior número de mulheres férteis.

Nesse sentido, apoiando-se em diversos estudos empíricos próprios e independentes, Buss e Kenrick (1998) e Buss e Schmitt (1993) afirmam que o homem deve ter desenvolvido mecanismos especializados para lidar com quatro facetas ou problemas adaptativos especializados: o número de parceiras, sua acessibilidade sexual, a identificação da fertilidade feminina e a evitação de compromisso e de investimento.

Esses mecanismos sexuais e/ou preferências psicológicas foram desenvolvidos de forma a tornar o homem capaz de aumentar o desejo de estar com um maior número de parceiras e a relaxar os padrões aceitáveis para essas parceiras de curto prazo (nos âmbitos de idade, inteligência, características pessoais e circunstâncias pessoais de estado conjugal), com restrições mínimas de tempo de conhecimento prévio para engajar em ato sexual. O homem deve ser capaz também de discriminar a acessibilidade, a fertilidade e o valor reprodutivo da mulher, através de sinais (sutis) e de dicas de fertilidade (oriundas de inferências acerca da idade e da saúde, presentes em características de aparência física e comportamento observável), e direcionar seu tempo, energia e recursos diretamente a ela, em detrimento de outras que demandem maior compromisso ou investimento anterior ao consentimento de sexo.

Os custos de uma mulher engajar-se em uma estratégia sexual de curto prazo são muito maiores que 
os dos homens. Além dos problemas enfrentados pelos homens, as mulheres ainda precisam lidar com danos à sua reputação social - que podem afetar suas chances em adquirir parceiros duradouros no futuro, devido à associação com uma maior incerteza de paternidade - e com abuso físico e sexual - devido à menor força física do sexo feminino (Bjorklund \& Kipp, 1996; Buss \& Kenrick, 1998; Buss \& Schmitt, 1993).

Há, entretanto, benefícios, como a possibilidade de obter melhor qualidade genética da prole; a utilização da estratégia de curto prazo como uma estratégia de avaliação de candidatos possíveis para relacionamentos duradouros; a extração imediata de recursos; e a obtenção de maior proteção, ao escolher homens com características sinalizadoras de provisão imediata de recursos, com maior força física e capacidade de impedir abusos, defendendo-a sem despender grande energia ou esforço, em detrimento daqueles que não se mostram muito propícios a fazê-lo.

\section{Estratégias sexuais de longo prazo}

A vantagem reprodutiva primordial para os homens em estratégias sexuais de longo prazo é a possibilidade de monopolizar a capacidade reprodutiva do curso de vida de uma mulher (Smuts, 1991, conforme citado por Buss \& Schmitt, 1993). Outros benefícios são a evitação dos custos de tempo, energia, curta duração do engajamento em estratégias de curto prazo e o proveito dos benefícios da mútua cooperação entre parceiros, da divisão do trabalho e do desenvolvimento de laços e alianças duradouras com a família da parceira, que permitem maior eficácia e economia, enquanto promovem o desenvolvimento da criança personificação da continuação do pool genético do casal (Buss \& Kenrick, 1998; Buss \& Schmitt, 1993).

Outras hipóteses acerca da procura de relações de longo prazo por parte do homem, já que maior sucesso reprodutivo é garantido através de uma estratégia de curto prazo, incluem a necessidade de obter parceiras com maior valor reprodutivo e promover melhor qualidade genética das crianças, pois, ao demonstrar sinais de compromisso duradouro, o homem pode obter uma parceira que não permita relações sexuais rapidamente e tenha melhores atributos fenotípicos ou genéticos (Buss \& Kenrick, 1998; Buss \& Schmitt, 1993), como também solucionar o problema da incerteza da paternidade, já que, devido à ovulação oculta da espécie humana, a permanência do homem ao lado da mulher constitui-se na única garantia de aumento da probabilidade de a prole ser realmente sua.

Após a escolha da parceira sexual, o ciúme sexual representa um mecanismo de manutenção da garantia de paternidade, ao funcionar como salvaguarda da parceira e como dissuasão de competidores intra- sexuais e, conseqüentemente, diminuir a probabilidade de inseminação da parceira por outro homem (Wilson \& Daly, 1995).

A lógica adaptativa da utilização de uma estratégia sexual de longo prazo para as mulheres reside no investimento parental masculino (provisão de alimentação e proteção própria e para sua prole, encontro e defesa de territórios, além de oportunidades de aprendizado e de transferência de status, poder, recursos e formação de fortes alianças recíprocas futuras para a sua prole) e no investimento em si própria, que encontra proteção e salvaguarda contra a desnutrição (Buss \& Kenrick, 1998; Buss \& Schmitt, 1993; Ellis, 1995). As preferências femininas quanto à escolha de parceiros devem ter evoluído na direção da resolução adaptativa de problemas frente à vantagem material imediata para a mulher e sua prole; a maior vantagem reprodutiva para a sua prole através da aquisição de benefícios sociais e econômicos - status; e a vantagem reprodutiva genética para sua prole, caso as variações na capacidade de aquisição de recursos sejam parcialmente hereditárias.

Haveria, assim, a valorização de parceiro/s mais ambiciosos, confiáveis, emocionalmente estáveis e menos promíscuos (Buss \& Schmitt, 1993). Buss (1995) acrescenta que, para realizar sua escolha, as mulheres baseiam-se em dicas, principalmente, ser trabalhador, ambicioso e inteligente, visto não ser completamente conhecido o potencial masculino em agregar recursos na época da reprodução.

Em suma, o poder masculino de atração deve ter evoluído em função da sua capacidade de prover recursos e investir tanto na própria mulher quanto na sua prole (Buss, 1995; Buss \& Kenrick, 1998; Buss \& Schmitt, 1993). O investimento parental servirá de guia para a escolha de parceiros por parte da mulher, quando o homem apresenta-se capaz e disposto a prover recursos.

Ellis (1995) adiciona um componente desenvolvimental à teoria: a atração sexual em cada cultura dependerá, para sua expressão, das experiências de cada indivíduo no curso de sua vida (ontogênese). $\mathrm{O}$ fator chave para a identificação e a determinação da qualidade do(a) parceiro(a) e, conseqüentemente, do seu investimento será a experiência. Não se pode esquecer ou denegar que são as estruturas psicológicas que permitem a extração de informação relevante e a sua utilização rápida e eficientemente para a solução de problemas adaptativos.

Nossos mecanismos psicológicos - especializados e direcionados para metas - permitem-nos aprender as coisas certas nas situações de aprendizagem tipicamente encontradas no nosso passado evolucionário [de caçadores-coletores]. Os mecanismos psicológicos subjacentes às percepções da atratividade 
sexual devem ser sensíveis às dicas ambientais que se correlacionam com o valor sexual do parceiro (Elis, 1995, p. 284).

Algumas condições, entretanto, são necessárias para que isto ocorra, tais como território no qual há (a) disponibilidade de reunir, defender e monopolizar recursos; (b) tendência de controle desses recursos por parte do homem; (c) relativamente alta variância na capacidade de reter recursos e na disponibilidade masculina de investi-los na mulher e na sua prole; e (d) suficiente valor reprodutivo feminino, capaz de atrair um parceiro investidor (Buss \& Schmitt, 1993).

Enfim, a lógica adaptativa de homens e mulheres varia em função do tempo empregado nas associações.

No caso de estratégias de longo prazo, as preferências adotadas por homens na escolha de uma parceira apontam para a resolução do problema da incerteza de paternidade, ao primarem pela monopolização da vida reprodutiva de uma mulher e pela escolha de uma parceira que se sobressaia pela fidelidade e pela lealdade sexual.

No caso de estratégias de curto prazo, como o objetivo primordial é a inseminação do maior número possível de parceiras, a escolha ocorreria de acordo com mecanismos de preferência de mulheres mais acessíveis sexualmente, a um menor custo de comprometimento e envolvimento de recursos.

Para as mulheres, supõe-se que, devido a um maior custo da estratégia de curto prazo, esta seja menos freqüente. Mesmo assim, ela seria orientada para o desenvolvimento de mecanismos psicológicos capazes de detectar parceiro/s com potencial de melhor carga genética, de possibilidade imediata de extração de recursos e de proteção contra abuso físico e/ou sexual de não-parceiros, isto é, parceiros fenotipicamente mais atrativos e ambiciosos. Esta estratégia serviria também como um dispositivo de avaliação de possíveis parceiros duradouros.

Nas estratégias de longo prazo, devem ter sido desenvolvidos mecanismos psicológicos de atração e detecção de parceiros dispostos e capazes de prover continuamente recursos e investimento parental para sua prole.

Até o presente momento, foram discutidos aspectos nos quais os mecanismos psicológicos de estratégias sexuais divergem entre homens e mulheres. Há, entretanto, similaridades, especialmente nas estratégias de longo prazo, presentes nos problemas de identificação de um aliado reciprocamente benéfico, de estabelecimento de compromisso e de identificação de parceiros com boas habilidades parentais.

A obtenção de vantagens reprodutivas das estratégias sexuais de longo prazo requer a identificação de um/a parceiro(a) confiável e cooperativo(a), com uma rede familiar, também aliada e cooperativa, para que a confluência de interesses seja maximizada. Em ambos os sexos, valorizam-se sinais de forte grau de compromisso do(as) parceiro(as) numa relação de longo prazo, cuja dica principal é a derrogação de outro(as) parceiro(as) em potencial em favor do(a) parceiro(a) atual.

Conforme Buss (1995), Buss e Schmitt (1993) e Ellis (1995), qualquer união de dois indivíduos geneticamente não relacionados tem o potencial de gerar conflitos, os quais serão manejados mais facilmente, através da concepção de uma prole, da monogamia e da inexistência de prole proveniente de associação sexual anterior. Ambos os sexos buscam parceiro(a)s com atributos similares, caracteristicamente compreensivos e gentis e com boas qualidades de parentalidade: inteligência, gentileza, educação, compreensão e estabilidade emocional. Segundo propõe Barkow (1989, conforme citado por Buss \& Schmitt, 1993), estes atributos são vistos como formas de melhor garantirem bons julgamentos acerca da proteção de crianças frente ao perigo; boas práticas de socialização; e capacidade de percepção de mudanças e tendências ambientais.

Além disso, diferenças individuais - dependentes de características pessoais de orientação sócio-sexual, ou seja, de propensão individual à adoção de estratégias de curto ou longo prazo - afetam o processo de escolha de parceiro(a)s (Snyder, Simpson \& Gangestad, 1986, conforme citado por Buss \& Schmitt, 1993). Estas diferenças supostamente persistiriam na sociedade em uma proporção dependente de freqüência, ou seja, como ajustamento às circunstâncias ecológicas, em que as estratégias adotadas por um indivíduo estão condicionadas às estratégias adotadas pelos outros.

Em nível ontogenético, fatores como idade e mudanças bruscas no estilo de vida (tais como divórcio, rápido aumento do status, melhora da saúde, diferença da proporção entre os sexos na população e fatores culturais) interferem na escolha da estratégia sexual a ser utilizada e permitem um trânsito provisório entre ambas.

\section{Críticas à Teoria de Estratégias Sexuais}

Hazan e Diamond (2000) retomam a Teoria de Estratégia Sexual de Buss e Schmitt (1993), considerada como uma extensão da Teoria de Investimento Parental de Trivers. Lembram que esses autores consideram a assimetria no investimento parental como uma solução para os problemas adaptativos frente a pressões ambientais, enfrentados na história evolucionária humana em prol de sucesso reprodutivo. Essa assimetria no investimento teria propiciado o desenvolvimento de mecanismos psicológicos específicos e diferenciados sexualmente. 
Hazan e Diamond (2000) concordam com a perspectiva teórica da existência de mecanismos psicológicos evoluídos para orientar o comportamento sexual de homens e mulheres. Discordam, contudo, quanto à existência de mecanismos específicos que funcionariam como estratégias de atuação sexual, designando quais qualidades seriam consideradas desejáveis e como ocorreria a seleção de parceiro(a)s com base nesses desejos inatos. Criticam igualmente a falha em explicitar e predizer o comportamento de seleção sexual na prática e a utilização de auto-relatos como estratégias inadequadas para a elaboração de uma teoria, já que o que se pensa ser o(a) parceiro(a) ideal, necessariamente não será o(a) escolhido(a).

Questionam ainda a teoria da estratégia sexual a partir de alguns pontos básicos (Hazan \& Diamond, 2000):

1. A existência de gatilhos específicos na seleção de parceiro(a)s seria desvantajosa para o sucesso reprodutivo, pois a obtenção do(a) parceiro(a) ideal nem sempre é possível;

2. Os ancestrais humanos viviam em bandos isolados. Não haveria grande possibilidade de escolha de parceiro(a)s e, portanto, não teria havido pressão para o desenvolvimento de mecanismos de escolha de parceiro(a)s baseados em diferenças sexuais;

3. A fertilidade e a capacidade de inseminação masculina, assim como a feminina, decrescem com a idade. Além disso, a expectativa de sobrevida de ambos os sexos não ultrapassava o seu período fértil;

4. Comparações com sociedades caçadoras-coletoras contemporâneas revelam que $90 \%$ dos jovens sexualmente maduros são férteis. Analogamente, não teria havido, portanto, um problema na escolha de parceiro(a)s férteis. Bastava-se evitar encontros sexuais com jovens que ainda não haviam atingido a puberdade e com aqueles que demonstravam sinais de envelhecimento ou de doença; e

5. O envolvimento feminino com grande número de parceiros poderia proporcionar uma melhor qualidade no pool genético da prole, visto que os diferentes tipos de esperma "lutariam" entre si para a fertilização do óvulo.

Considerando-se esses elementos, o sistema de escolha sexual deve ter sido desenhado de forma a equipar os indivíduos para se afastarem de escolhas fatais e não especificamente para aproximá-los de escolhas ideais (Hazan \& Diamond, 2000).

\section{Uma proposta alternativa: apego e escolha de parceiro(a)s sexuais}

Hazan e Diamond (2000) propõem como norma para o padrão de acasalamento de homens e mulheres a formação de vínculos entre o(a)s parceiro(a)s sexuais. Com base na teoria de apego de Bowlby, "propensão, familiaridade e enamoramento romântico são propostos como mecanismos que explicam como as 'escolhas' sexuais atuais são realizadas e como relacionamentos sexuais são estabelecidos" (p. 188).

O nascimento de bebês muito imaturos, em decorrência de mudanças na postura de locomoção, proporcionou maior sobrevivência para as crianças humanas, maior plasticidade neural pós-natal e maior potencial de aprendizagem. A contrapartida em custos foi o maior investimento parental. Nesse sentido, a prematuridade infantil e a conseqüente necessidade de maior tempo e esforço parental na proteção, no cuidado e na socialização das crianças demandaram o desenvolvimento de um mecanismo de ligação entre criança e cuidador - nesse caso, o apego.

Do mesmo modo, esses mesmos fatores exigiram uma maior ligação entre o(a)s parceiro(a)s reprodutivo(a)s. Hazan e Diamond (2000) propõem que a exaptação do mecanismo de apego - cooptação de uma estrutura evoluída para um propósito novo - teria sido utilizada para a manutenção do relacionamento entre casais.

A relação estabelecida entre parceiros (Hazan \& Diamond, 2000) segue o princípio do apego (Bowlby, 1990a), a saber: (1) manutenção de proximidade: busca e manutenção de proximidade física; (2) abrigo seguro: busca de conforto e auxílio quando necessário; (3) ansiedade de separação: experiência de ansiedade frente a separações inesperadas ou prolongadas; e (4) base segura: confiança na figura de apego como uma fonte de segurança através da qual se pode envolver em atividades exploratórias e não relacionadas ao apego.

Fatores como a seqüência protesto, desespero e desapego e o aumento significativo de riscos por vários problemas de saúde físicos e mentais, vistas nas reações diante de situações de separação e perda nos relacionamentos maritais; em contrapartida com outras formas de relacionamento social (Bowlby, 1990b); a segregação de hormônios semelhantes no relacionamento entre cuidador/criança e entre parceiros românticos; a anatomia e a fisiologia sexuais que permitem a ocorrência de relações sexuais em qualquer período do ciclo reprodutivo feminino e em posições sexuais demandantes de maior proximidade emocional; e a busca das mesmas características (bondade, compreensão e inteligência) nas escolhas sexuais de ambos os sexos (Buss, 1995; Buss \& Schmitt, 1993; Ellis, 1995; Hazan \& Diamond, 2000) corroboram a afirmação da existência de apego nas relações entre parceiros sexuais. 
Hazan e Diamond (2000) apresentam vários estudos independentes que apoiam o estabelecimento de apego entre parceiros como uma forma de sucesso reprodutivo, pois se consegue combinar três desafios adaptativos: sobrevivência até a idade reprodutiva, aquisição e permanência de um/a parceiro(a) e promoção de cuidado adequado para a prole, de forma que esta também alcance a idade reprodutiva.

$\mathrm{O}$ estabelecimento de relacionamentos duradouros também contribuiria para o sucesso reprodutivo ao promover maior regularidade às ovulações; sentimentos de pertença, apoio, segurança e defesa frente a ameaças; e maior chance à prole de estabelecer relações igualmente duradouras (Hazan \& Diamond, 2000). Acrescenta-se a isto a ocorrência de excitação física, idealização do(a) parceiro(a) e a íntima relação da liberação de anfetamina corpórea no processo de enamoramento.

Esses fatores são significativos para a consideração do apego como adaptação evolutiva para o sucesso reprodutivo de ambos os sexos, indistintamente. Mesmo assim, não se postula uma escolha sexual de parceiro(a)s aleatória. Descarta-se, porém, uma escolha estratégica. Considera-se a propensão como fator da escolha. A gama de escolha de parceiro(a)s “(...) consiste naqueles indivíduos que, concomitantemente, superam os critérios de rejeição de um determinado indivíduo e vivem, trabalham ou divertem-se nas proximidades" (Hazan \& Diamond, 2000, p. 196). Há uma escolha segundo o critério de avaliação custo/benefício no qual o indivíduo avalia o próprio valor sexual e, posteriormente, avalia o valor sexual do(a) parceiro(a) em potencial.

$\mathrm{O}$ fator de propensão reside na atração adequada e na acessibilidade imediata. Ademais, a familiaridade aumentaria o conhecimento das características individuais e a propensão à escolha e a atração de determinado indivíduo.

Familiaridade, propensão, preferências e qualidades individuais reduzem consideravelmente a gama de escolhas. A determinação de um/a parceiro(a) específico(a), contudo, ocorre através do enamoramento romântico. Este se dá por meio da percepção da reciprocidade do gostar, que se retroalimenta na expressão de comportamentos mútuos de busca de proximidade física. "Para um número significativo, apenas isto é suficiente para capturar e manter seus interesses até que uma relação de apego mais forte se desenvolva" (Hazan \& Diamond, 2000, p. 197), laço este que necessitará de aproximadamente dois anos (seis meses a mais ou a menos) - enquadre temporal correspondente à duração do enamoramento romântico.

Essa visão é compatível com a perspectiva adotada por Keller (1996), que busca ligar biologia e cultura através dos padrões de apego estabelecidos nos anos iniciais.

Nessa abordagem, derivada primariamente da teoria do investimento parental da Biologia Evolucionária, as circunstâncias ecológicas em que o indivíduo vive influenciarão suas estratégias de investimento parental.

Essa influência ocorre indiretamente, através dos padrões de apego desenvolvidos nos primeiros anos de vida, de modo que o estilo de investimento parental mais quantitativo, com baixo cuidado despendido a cada indivíduo da prole, trará, como conseqüência, o desenvolvimento de um vínculo de apego inseguro. Ao contrário, um estilo de investimento parental mais qualitativo, caraterizado por uma grande quantidade de cuidado dispensado a cada filho, produzirá, em geral, relações de apego seguras (Keller, 1996).

Esse vínculo inicial afetará os padrões de reprodução na idade adulta, tanto na formação de pares, quanto nas decisões reprodutivas e de cuidado à prole.

\section{CONSIDERAÇÕES FINAIS}

Neste artigo, foram apresentadas duas propostas de explicação para o processo de escolha sexual de parceiro(a)s. Uma delas evoca a existência de uma estratégia como mecanismo de escolha - o investimento parental. A outra prioriza a formação de vínculos entre os parceiros.

Devem-se analisar criticamente as propostas de explicação do funcionamento psicológico humano. Como se compreender mecanismos de preferência sexual de parceiro(a)s e investimento parental? Prevalecem estratégias? Propensões?

No estado atual da pesquisa na área, não há evidência empírica suficiente para refutar uma dessas visões ou mesmo ambas. Respostas conclusivas serão produzidas testando-se hipóteses rivais baseadas em diferentes visões.

É possível, todavia, que uma terceira perspectiva unifique as duas teorias, ao integrar questões causais, funcionais, ontogenéticas e filogenéticas. Uma teoria de estratégia poderia ser compatível com a teoria de vínculo, como na visão proposta por Keller (1996). Nessa abordagem, busca-se um vínculo entre base biológica (interesses reprodutivos) e a cultura, o que deve ser estudado no ciclo de vida completo do indivíduo. Os esforços reprodutivos dos indivíduos estariam relacionados com seus esforços somáticos. Embora a espécie humana seja, por excelência, uma espécie de alto investimento parental, a variabilidade existente na quantidade de investimento parental alocado à prole teria o potencial de influenciar dois estilos reprodutivos distintos, representando trajetórias desenvolvimentais diferenciadas. 
O tipo 1 descreve o indivíduo que, na infância, experimenta um ambiente de escassez de recursos, com baixo investimento parental e clima familiar insatisfatório. Situações típicas desse ambiente podem incluir ausência paterna, conflitos conjugais, negligência e violência familiar. No outro extremo, o tipo 2 descreveria o indivíduo que dispõe de recursos materiais satisfatórios e experimenta um alto investimento parental e um clima familiar positivo (Keller, 1996).

Não só essas diferenças em experiência inicial teriam impacto nas relações de apego construídas com os pais durante os primeiros anos de vida, como também conduziriam a consequiências de longo prazo, as quais se estenderiam por todo o ciclo de vida, a começar por diferenças no timing da puberdade, com o tipo 1 amadurecendo mais cedo que o tipo 2. Em seguida, os estilos reprodutivos continuariam a se diferenciar, resultando num estilo mais quantitativo para o tipo 1 (mais filhos, menor espaçamento entre os nascimentos e baixo investimento parental) e mais qualitativo para o tipo 2 (menos e mais espaçados filhos e alto investimento parental).

Em qualquer caso, a teoria deveria explicar, simultaneamente, universalidade, diversidade cultural e trajetórias desenvolvimentais particulares, à luz da perspectiva evolucionária. Esse empreendimento não pode ser alcançado sem que sejam elucidados os processos psicológicos básicos que controlam inclinações e preferências (dimensão causal), os benefícios reprodutivos de uma ou outra estratégia reprodutiva (dimensão funcional), a origem e o desenvolvimento dos processos psicológicos no curso da vida do indivíduo (dimensão ontogenética) e as condições prevalecentes no ambiente evolucionário da espécie, as quais exerceram pressões seletivas em direções específicas (dimensão filogenética).

Enquanto essa teoria abrangente não emerge, e até como uma contribuição para a sua construção, a Psicologia do Desenvolvimento pode desempenhar um papel decisivo na elucidação dos mecanismos responsáveis pelo comportamento reprodutivo e parental, o que vem sendo feito nos estudos sobre apego adulto e suas conexões desenvolvimentais.

Estudar a evolução do apego durante o ciclo de vida e suas relações com os estilos parentais na vida adulta é um ramo da Psicologia Evolucionária do Desenvolvimento que pode trazer uma valiosa contribuição para a área. Além disso, o campo de estudo pode trazer uma contribuição imediata e socialmente relevante, na medida em que pode orientar políticas públicas relacionadas com a promoção de vínculos primários na infância, decisões sobre onde e como cuidar da criança, a organização da educação, políticas de proteção aos interesses da criança em casos de conflitos parentais, dentre outros.

\section{REFERÊNCIAS}

Bjorklund, D. F. \& Kipp, K. (1996). Parental investment theory and gender differences in the evolution of inhibition mechanisms. Psychological Bulletin, 120, 163-188.

Bowlby, J. (1990a). Apego: apego e perda. São Paulo: Martins Fontes.

Bowlby, J. (1990b). Apego: perda, tristeza e depressão. São Paulo: Martins Fontes.

Buss, D. M. (1995). Mate preference mechanisms: consequences for partner choice and intrasexual competition. Em J. H. Barkow, L. Cosmides \& J. Tooby (Orgs.), The adapted mind: evolutionary psychology and the generation of culture (pp. 249-266). Oxford/London: University Press.

Buss, D. M., Kenrick, D. T. (1998). Evolutionary social psychology. Em D. T. Gilbert, S. T. Fiske \& G. Lindzey. (Orgs.), The handbook of social psychology (pp. 982-1026). New York/ Oxford: Oxford University Press.

Buss, D. M. \& Schmitt, D. P. (1993). Sexual strategies theory: an evolutionary perspective on human mating. Psychological Review, 100, 204-232.

Ellis, B. J. (1995). The evolution of sexual attraction: evaluative mechanisms in women. Em J. H. Barkow, L. Cosmides \& J. Tooby (Orgs.), The adapted mind: evolutionary psychology and the generation of culture (pp. 267-288). Oxford/London: University Press.

Geary, D. C. \& Flinn, M. V. (2002). Sex differences in behavioral and hormonal response to social threat: commentary on Taylor et al. (2000). Psychological Review, 109, 745-750.

Hazan, C. \& Diamond, L. M. (2000). The place of attachment in human mating. Review of General Psychology, 4, 186-204.

Keller, H. (1996). Evolutionary approaches. Em J. W. Berry, Y. H. Poortinga \& J. Pandey (Orgs.), Handbook of cross-cultural psychology (pp. 215-256). Boston: Allyn and Bacon.

Kriegman, D. (1999). Parental involvement, sexual selection, and evolved mating strategies: implications for psychoanalysis. Psychoanalytic Psychology, 16, 528-553.

Wilson, M. \& Daly, M. (1995). The man who mistook his wife for a chattel. Em D. T. Gilbert, S. T. Fiske \& G. Lindzey. (Orgs.), The handbook of social psychology. New York/Oxford: Oxford University Press (pp. 289-322). Oxford/London: University Press.

\section{Sobre as autoras:}

Enviado: 11/10/2004 Revisado: $14 / 02 / 2005$ Aceito: 20/02/2005 
Roberta Tavares de Melo Borrione: Mestra em Psicologia pela Universidade Federal da Bahia (PPGPSI - UFBA/Bolsista CAPES). Endereço eletrônico: robborrione@yahoo.com.br.

Eulina da Rocha Lordelo: Doutora em Psicologia. Professora da Universidade Federal da Bahia. Pesquisadora CNPq. Endereço eletrônico: eulina@ufba.br. 